Archived version from NCDOCKS Institutional Repository http://libres.uncg.edu/ir/asu/

Curtis, Andrew, Bin L., B. D. Marx, J. W. Mills and J. Pine (August 16, 2010). A multiple additive regression tree analysis of three exposure measures during Hurricane Katrina. Disasters: The Journal of Disaster Studies, Policy and Management. Vol 35 (1) pp. 19-35. [ISSN: 0361-3666] The version of record is published by Wiley and can be accessed at http://onlinelibrary.wiley.com/doi/10.1111/j.1467-7717.2010.01190.x/pdf

\title{
A Multiple Additive Regression Tree Analysis of Three Exposure Measures During Hurricane Katrina
}

\author{
Andrew Curtis, Bin Li, Brian D. Marx, \\ Jacqueline W. Mills and John Pine
}

\begin{abstract}
This paper analyses structural and personal exposure to Hurricane Katrina. Structural exposure is measured by flood height and building damage; personal exposure is measured by the locations of 911 calls made during the response. Using these variables, this paper characterizes the geography of exposure and also demonstrates the utility of a robust analytical approach in understanding health-related challenges to disadvantaged populations during recovery. Analysis is conducted using a contemporary statistical approach, a multiple additive regression tree (MART), which displays considerable improvement over traditional regression analysis. By using MART, the percentage of improvement in R-squares over standard multiple linear regression ranges from about 62 to more than 100 per cent. The most revealing finding is the modelled verification that African Americans experienced disproportionate exposure in both structural and personal contexts. Given the impact of exposure to health outcomes, this finding has implications for understanding the long-term health challenges facing this population.
\end{abstract}




\section{Introduction}

Hurricane Katrina2 made landfall along the Louisiana and the Mississippi Gulf Coast in the morning hours of 29 August 2005. The resulting storm surge and breaches to the New Orleans levee system resulted in devastation on an unprecedented scale for a major North American city. Although the disproportionate impact of this disaster has been discussed in multiple forums, especially with reference to the AfricanAmerican population, these discussions have largely been based on descriptive analyses, cartographic overlays and the matching of exposure variables to census information (Curtis, Mills and Leitner, 2007; Green et al., 2007; Sharkey, 2007). However, in order to develop models of recovery in general, as well as to theorise about the ongoing recovery situation of Orleans and St Bernard Parishes, a more rigorous analytical frame is required. Indeed, a recent paper by Green et al. (2007) suggests that the exaggerated description of damage in the Lower 9th Ward by the media had implications in the lack of recovery for that neighbourhood. This accusation was expanded by presenting the results of a damage assessment to that area carried out by field teams, which found far less serious structural damage. Whether or not the authors are correct in their interpretation of the amount of damage, the issue to be taken from this and other papers is that there is still relatively little in terms of replicable, modelled investigations of exposure to the events of Katrina.

This paper adds rigour by using a contemporary statistical approach, a multiple additive regression tree (MART), which has the advantage over traditional regression models in that it fits locally and adaptively without assuming any parametric form for spatial dependency among the input variables. Three measures of exposure are analysed against variables selected for their importance in predicting subsequent health vulnerability, which is a consideration in the successful recovery of the communities. Two of the measures of exposure involve structural characteristics (flood height and building damage) one of which (flooding) has been a frequently chosen measure in previous analyses of the storm's impact (Sharkey, 2007). Both metrics are used in order to capture the full extent of structural exposure to wind and water. Due to the attention given to flooding, it is often forgotten that some areas did not flood, but did experience severe damage from hurricane winds. In order to capture personal exposure to the storm, a third exposure, 911 calls generated during the first three days after the storm, is also analysed. Examining both structural and personal aspects provides a novel and more comprehensive approach to measuring exposure than simply looking at flooding alone.

\section{Modelling exposure and recovery related census variables}

The analysis employed in this paper analyses three measures of exposure against variables known to be associated with post-disaster health vulnerability. These include the degree to which flooding occurred, which serves as an impediment to recovery given that the deeper the flood, the greater the chance of losing the home or of it requiring substantial clean-up and re-building efforts. A second measure, the degree of damage to the home, also captures structural exposure, but takes into 
account that serious wind damage could occur in areas with little or no flooding. The third measure, the number of 911 calls for the first three days after landfall, is a proxy for presence during the storm and the subsequent levee breaches. This type of exposure indicates the possibility that this person experienced physical health burdens such as injury, dehydration and exacerbation of chronic illness. Mental health consequences of personal exposure include experiencing the physical health burdens of family members and neighbours, in addition to their death, and also potentially dealing with crime and feelings of abandonment.

Previous investigations of exposure have lacked sophistication, relying only on map overlay and descriptive comparison among census variables. For example, Sharkey's (2007) analysis of three measures of exposure (mortalities, missing individuals and flood height) found that although the elderly comprised the majority of mortalities within Orleans Parish, African-American deaths within this cohort were higher than expected given the pre-Katrina racial distribution of those aged 65 years and older. In this paper, geocoded events (deaths) and flood heights were overlaid on a census map. Though revealing, this approach lacks rigour in terms of providing a model base onto which other hypotheses can be postulated. For example, Sharkey comments, 'Though the map seems to suggest that more bodies were found in Black areas of the city, it does not demonstrate this conclusively' (2007, p. 492). In order to move forward and discuss subsequent issues resulting from this catastrophe, as well as determine the actual nature of the exposure associations, a more rigorous statistical analysis is required to act as a base upon which impediments to neighbourhood recovery in 2008 can be theorised.

\section{Methodology}

\section{Measures of acuteness of exposure}

Katrina caused catastrophic damage to residential, commercial and industrial property and resulted in the deaths of more than 1,500 people in Orleans Parish, which demonstrates the extent of both structural and personal exposure. There are different ways to measure the physical impact of the storm on a neighbourhood, but for this analysis flood height (Flood) and damage to buildings (Damage) were chosen. Although approximately 80 per cent of New Orleans was under water on 2 September 2005 , the depth of floodwater varied across the city. This depth was largely as a result of three factors; proximity to a levee breach, inundation from storm surge, and building height below sea level. Flooding can cause damage by the force of water, the duration of the event, and the height of floodwater. The last of the three measures is used in this analysis as it captures elements of the other two; greater flood heights are partly due to proximity to the flood focus and, ceteris paribus, the deeper the flood the longer the waters will remain. 
Figure 1 displays a map of average flood depth (in feet) by census tract on 2 September 2005. These data are aggregated from a depth grid composed of $25 \mathrm{~m} 2$ cells, which reflect high-water marks recorded by the US Army Corps of Engineers and their contractors throughout the City of New Orleans. In order to match spatially the flood grid with the indicators used in the analysis, the flood grid was aggregated to a census tract coverage by calculating the average flood depth based on cells falling inside each census unit.

Figure 1 Water depth on 2 September 2005

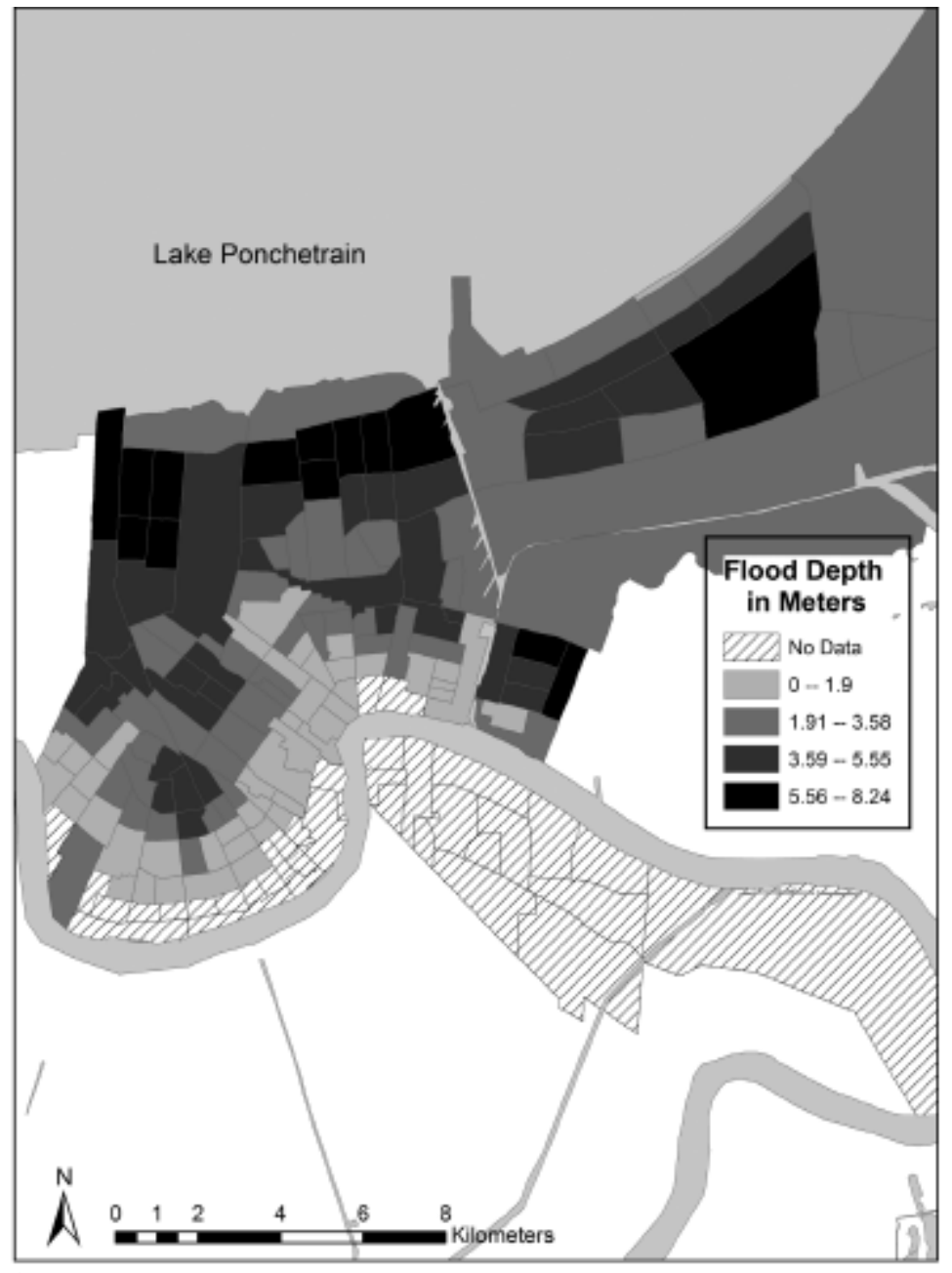

Source: Cartography by the authors. High-water marks that were recorded by the US Army Corps of Engineers and their contractors throughout the City of New Orleans have been manipulated into census tract enumeration units by the authors.

The second measure of structural exposure was a comprehensive damage assessment of residential, commercial and industrial buildings undertaken by FEMA in the weeks following Katrina. The variable chosen for inclusion was a manipulation of the percentage of damage for each building. For each address in a census tract the percentage of buildings suffering greater than 50 per cent damage (chosen to reflect severe structural impact and therefore an impediment to recovery) was calculated. In order to remove the problem associated with areas containing relatively 
few structures, or if an area had been inadequately sampled, only census tracts with more than 50 buildings were included in the analysis (resulting in 152 of 181 total tracts). Although this measure is similar to the damage caused by flooding, it captures the additional destruction caused by wind and even fire. It is also more accurate in terms of delivering point-specific damage assessment of a property that a) captures the physical 'home loss' experienced by the individual based on the ability of the property to survive the storm, which in turn b) captures an impediment to recover.

A third measure of storm exposure was included in the analysis in order to capture personal exposure to the storm. 911 calls (911) originating from Orleans Parish for the period of 29-31 August were obtained from the City of New Orleans 911

Communication Center (Figure 2). Data include the phone number and address of the caller, contact information of the individual needing assistance, and two columns of emergency attributes-for example, any specific medical needs of the caller. Each

Figure 2911 call locations

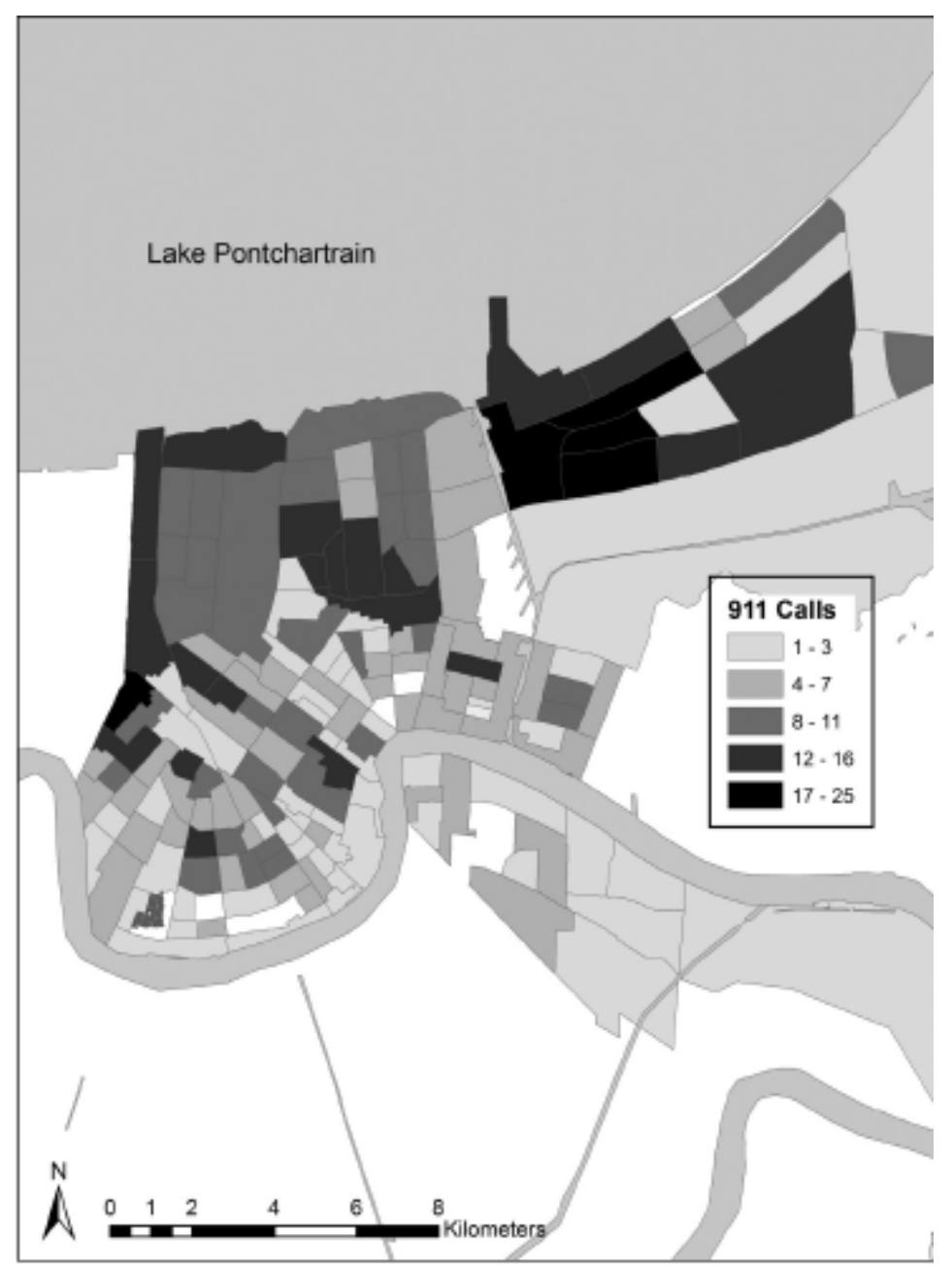

Source: 911 calls originating from Orleans Parish for the period of 29-31 August. Data from the City of New Orleans 911 Communication Center and modified for mapping purposes by the authors. 
call was also weighted by the total number of people mentioned in the message, though calls relating to more than 20 people were removed due to their institutional nature (prisons, hospitals) rather than residential association. Therefore, the resulting variable includes the total number of people needing 911 assistance per census tract. As far as the authors are aware, this measure has not previously been used in an analysis of Katrina exposure, nor any other post-disaster analysis of this type.

\section{Measures predicting the potential for recovery impediments}

Five socio-economic indicators were extracted from the US Census Bureau 2000 Summary File 3 (SF3) for 181 tracts. Two of these indicators were selected because of their previous associations as risk factors for psychopathology and the chronic health burden of the urban poor, particularly in New Orleans. These variables are 1) the proportion of African Americans in each census tract (AfAm) and 2) the percentage of families with a female householder, no husband present, with related children under 18 years (FemHead). Then, one variable was selected as a proxy for the lack of choice in having to face the storm: the percentage of occupied housing units with no vehicles available (NoCar). The final two variables were selected because of potential impediments to recovery both on a personal level and with implications for the neighbourhood. These characteristics are 1) the percentage of persons 25 years and over with less than nine years of school (Edu), as lower education levels have previously been linked to neighbourhood risk resulting in stress and subsequent health problems, and 2) the percentage of households who are renters (Rent). Additionally, a final variable for inclusion was a generalised measure of the pre-event disease burden, sexually transmitted disease (STD). Areas of Orleans Parish with no population (City Park and New Orleans East) were removed from the analysis. The following section provides a more detailed justification for the inclusion of each variable in the model.

\section{African-American neighbourhoods}

The social, political and urban infrastructure challenges faced by many AfricanAmerican neighbourhoods often result in overall poorer health (Satcher al., 2005), which in New Orleans includes a high chronic (for example, diabetes and hypertension) and infectious disease burden (for example HIVIAIDS). In terms of psychopathology African Americans are known to suffer disproportionately high neighbourhood and social stressors (Hambrick-Dixon, 2002; Schulz et al., 2000). African Americans in the United States also have the highest risk for low birth weight deliveries, short gestation births and infant mortality (Lane et al., 2001). This is especially true for African Americans in Louisiana (Curtis and Leitner, 2006). Research has also shown that the density or isolation of African-American families within a neighbourhood can likewise affect health outcomes (Pickett et al., 2005; Roberts, 1997).

The hazards literature frequently comments on the risks minorities face, which include living in the most physically vulnerable areas (Peacock et al., 2000; Pulido, 
2000), suffering through culturally and ethnically insensitive response strategies (Klitzman and Freudenberg, 2003; Phillips, 1993), and a combination of factors resulting in an unwillingness to evacuate (Howell, 2005). Therefore, it is possible that African Americans in Orleans Parish were more likely to have suffered the greatest exposure, while at the same time carrying pre-existing health conditions that would be further intensified by that exposure.

\section{Female-headed families with children in the home}

Females are often identified as being vulnerable to the effects of a disaster (Cutter et al., 2003; Enarson, 2002). They are also a population of concern in terms of suffering disproportionate post-disaster health outcomes (Norris et al., 2002). Initial Katrina mental health investigations for the Orleans Parish area demonstrate this situation (DeSalvo et al., 2007; Galea et al., 2007). However, the addition of children, especially if a single mother is the head of household, introduces even further social vulnerability and health risk potential (Greenough et al., 2008; Morrow, 2002).

Figure 3 shows a nursery sign from the 8th Ward. The flood height from Katrina is clearly visible, as is the pick-up hour of $1 \mathrm{am}$, which suggests a population comprised of single parents (probably mothers) who also have work obligations. The typical fear and uncertainty of events unfolding after a disaster will be magnified in a single mother, who is likely to be removed from the previously relied upon family and local community support. In addition, social services that are likely to have played a large role in supporting the family will also have been disrupted (Kaniasty and Norris, 1995; Weisler et al., 2006). The state of the mother's mental health and negative coping mechanisms are closely tied to those of her children (Abramson and Garfield, 2006).

Figure 3 Flood height and pre-Katrina nursery hours

The health of the children is

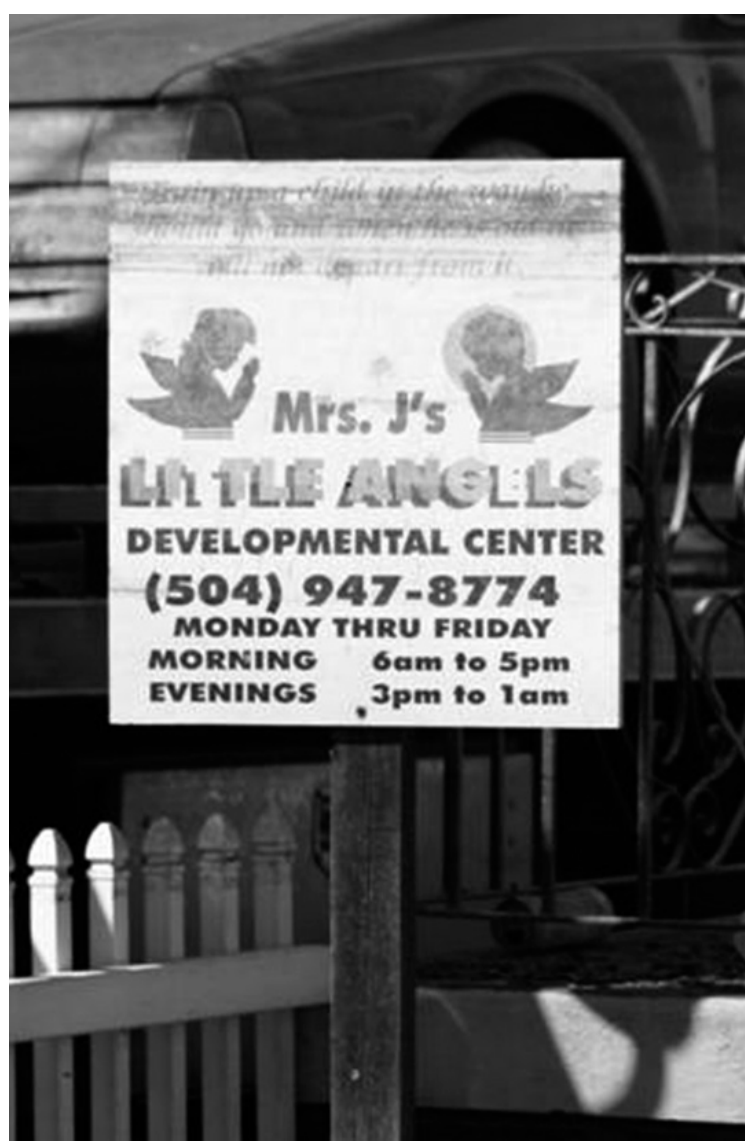

Photo: Andrew Curtis (October 2005). also of concern, as they are more susceptible to dehydration and malnutrition and have a less well-developed immune system. Therefore, in combination with poorer sanitary skills due to age, they are also more susceptible to infectious 
disease outbreaks (Dolan and Krug, 2006). Furthermore, post-traumatic stress disorder in children can lead to physical changes in the brain, resulting in problems following the child into adulthood (Dolan and Krug, 2006). In addition, if the female head-of-household caregiver is either pregnant, or became pregnant in the months afterwards (which occurred frequently in the temporary living environments following the storm), the risk to a successful pregnancy would be extremely high. If one assumes all women of childbearing age are of equal likelihood of becoming pregnant, and without more specific birth information, the geography of this variable should generate the most concern.

\section{Lack of transportation}

It has been estimated that up to 250,000 residents of New Orleans were reliant on the city's inadequate bus provision prior to Katrina (Bourque et al., 2006). However, there are other reasons why people do not evacuate from a hurricane, including evacuation fatigue, owning pets, not enough money to pay for motel rooms, and not having a geographically extensive network of friends and relatives who could temporarily provide shelter (Dow and Cutter, 1998, 2000, 2002; Wolshon, Hamilton et al., 2005; Wolshon, Urbina et al., 2005). The inclusion of a lack of transportation not only captures the increased exposure in terms of having choice removed, but also as a proxy for lack of mobility and access to services pre-Katrina, which in turn suggests a reliance on community for support.

\section{Education}

Individuals with a lower educational attainment are less likely to navigate successfully through the systems of relief, insurance and compensation, which in turn can lengthen the exposure of the disaster, resulting in both a prolonged refugee period and a spatially uneven residential return. In addition, a more poorly educated cohort may have less ability to recognise developing symptoms needing medical attention. Similarly, a lack of education may impact their health behaviours-for example, if the individual is aware of how some coping strategies might affect a foetus.

\section{Renters}

A high proportion of rental properties may capture the degree to which a neighbourhood is in flux, with renters being more transient and more open to relocation. Therefore the proportion of renters in a census tract may have future implications to community level mental health outcomes as owner-occupied residences will be rebuilt and reoccupied first, resulting in a potential mixing of returnee and abandoned properties. Returnees in such neighbourhoods will be left with greater uncertainty as to when the community will re-establish. As mentioned before, this has implications for both psychopathology and birth outcomes. In addition, if rental properties lag behind in terms of rebuilding, this could result in a neighbour-toneighbour spillover of health concerns such as mould toxicity and vermin presence.

Characteristics of the residential situation, including distance to work from the 
new home, comparison of the new compared to old residence, damage to the home, and living in a cramped temporary trailer, have all been included as potential stressors in different post-Katrina health investigations (Abramson and Garfield, 2006;

DeSalvo et al., 2007; Galea et al., 2007; Rath et al., 2007).

\section{Sexually transmitted diseases}

In addition to the previously described census variables commonly associated with health vulnerability, a crude measure of the pre-Katrina disease burden is also included, the gonorrhea rate per census tract (STD) classified into deciles (by the Louisiana State Office of Public Health in order to preserve confidentiality).

\section{Statistical analysis}

The statistical approach employed to investigate the relationship between exposure and potential recovery is closely related to regression tree analysis (Breiman et al., 1984). Tree-based models provide an alternative to linear and additive models for regression problems (Breiman et al., 1984). The models are fitted by a recursive partitioning whereby a dataset is successively split into increasingly homogenous subsets until the information gained by additional splits is not out-weighed by the additional complexity due to the tree's growth. Regression trees are adept at capturing non-additive behaviour, for example, interactions among independent variables are routinely and automatically handled. Further, regression tree analysis can easily handle a mix of numeric and factor independent variables.

Figure 4 Regression tree for 181 census tracts in Orleans Parish

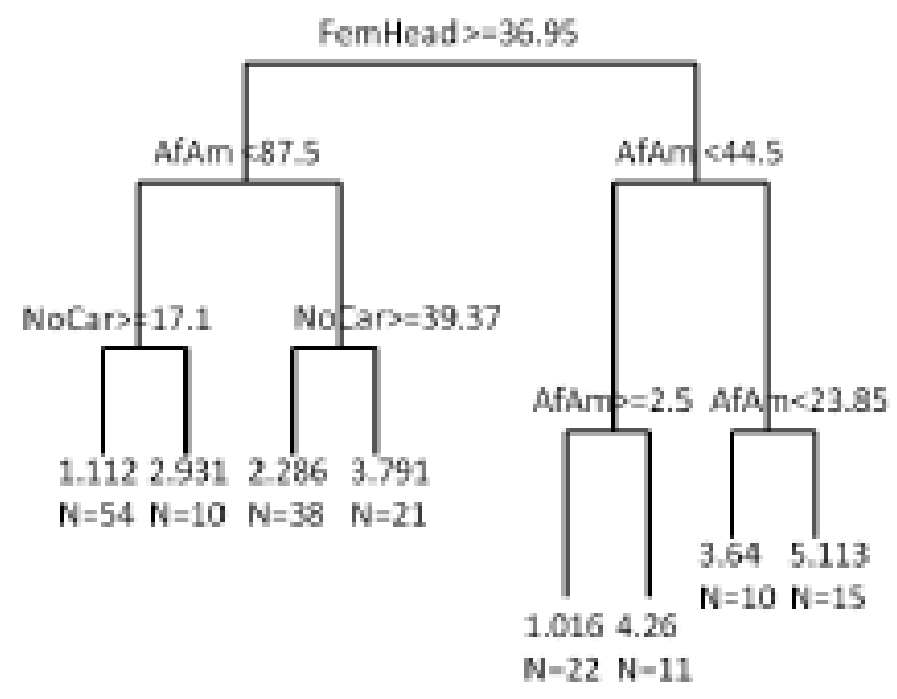

Source: Authors' elaboration of results. 
Figure 4 shows a regression tree with eight leaves (terminal nodes). For example, the leftmost leaf, which covers 54 census tracts, indicates the average water depth for the tracts with 'FemHead $\geq 36.95$ per cent' (the percentage of female head-ofhousehold families being greater than 36.95), 'AfAm<87.5 per cent' (the percentage of African Americans being less than 87.5) and 'NoCar $\geq 17.1$ per cent' (the percentage of families with no vehicles being greater than 17.1) is 1.112 feet. Thus the 54 census tracks with moderate to high single families ( $>37$ per cent), with a racial distribution of less than 88 per cent African-American residents, and with moderate to high no-vehicle families had an average flood level of about 1.112 feet.

However, one of the major problems with this type of tree approach to data analysis is in the instability of tree-structures-that is, a small change of input data will alter the entire tree structure, which is a crucial obstacle to more sound interpretability. Therefore, MART, an improvement on the traditional tree analysis originally proposed by Friedman (2001), was employed for this study. Unlike conventional regression based techniques, MART fits a large number of relatively simple regression trees, whose predictions are then combined to give more stable estimates of the response. Specifically, MART uses an iterative method for developing a final model in a forward stage-wise fashion, progressively adding regression trees to the model, while following the maximal gradient descent direction. MART not only overcomes the instability from a single regression tree, but also achieves exceptionally accurate prediction as well as advanced interpretability. Due to its ability to fit interaction effects and nonlinear relationships between a response variable and its predictors, MART is advantageous when analysing the complex relationships typically found in social science datasets. In this paper, MART is run by using the gbm library (Ridgeway, 2006) in R (version 2.0.1), a freely available statistical software.

Fitting a MART model requires specification of two main parameters: learning rate and the size of the individual trees. The learning rate controls the rate at which model complexity is increased, with smaller values resulting in the fitting of a larger number of trees, each of lower individual influence and generally giving superior predictive performance (Friedman, 2001). The size (number of splits) of the individual trees is controlled by a parameter called maximum interaction depth in the $\mathbf{g b m}$ library. A value of this parameter of one indicates that each tree consists of a single node (or two leaves).

\section{Results}

The focus of the analysis is on the link between population characteristics of health and social vulnerability and three dependent variables as measures of an acute Katrina exposure: 1) flood depth denoted as 'flood'; 2) percentage of buildings with greater than 50 per cent damage denoted as 'damage'; 3) total number of 911 calls up until 31 August 2005 denoted as '911'. The above three exposure variables were modelled 
on the six previously mentioned social characteristics. Using these variables in quantitative analysis, this paper investigates the questions of who was most exposed

Table 1 R-squares in MLR and MART

\begin{tabular}{|c|c|c|c|}
\hline Depandant varisblo & Food & Danagg & 911 \\
\hline MLR & 0.1619 & 0.2557 & 0.1506 \\
\hline MART & 0.3900 & 0.4170 & 0.3141 \\
\hline Percantage improvenent & 103.8 & a.ce & 100.5 \\
\hline
\end{tabular}

to Katrina, what are the implications for recovery of these people and, specifically, what does this exposure portend for their long-term health?

A variety of statistical methodologies were applied including standard multiple linear regression (MLR) and the more contemporary statistical approach, MART. Empirical results have shown that MART is a powerful nonparametric alternative to standard MLR. In comparison to MLR, MART has advantages, inherited from regression trees, in dealing with nonlinearity, interactions, mixed-type of inputs and missing values. To give an idea of gains for using MART over MLR, refer to Table 1. We find the percentage of improvement in R-squares ranges from approximately 63 per cent to 108 per cent. The detailed results for modelling each of the three dependent variables are presented in the following three subsections. In the model fitting we fixed the learning rate at 0.001 and the maximum depth of tree at four.

\section{Modelling flood depth}

The proportion of variability explained by the final MART model (or R-square) is approximately 33 per cent, where the final MART model consisted of 1,167 individual regression trees. It is useful to understand the relative importance or contribution of each explanatory variable in association with the response variable. The left panel of Figure 5 shows the relative variable importance on the associative variables. Notice that the summation of the variable importance for all the variables totals 100 per cent. Among the eight vulnerability indicators, AfAm and FemHead are the two most influential appearing in the model. The variables NoCar and Edu have moderate importance, while Rent and STD have the least importance.

After establishing the relative importance for each variable, the nature of the dependence of the fitted model on any subset of explanatory variables is of interest. The partial dependence function, introduced by Friedman (2001), can be used to examine graphically the dependence of a fitted model on low cardinality subsets of the variables. Figure 5 shows the partial dependence plots for the two most important variables: AfAm and FemHead. Both variables display non-linear effects on the fitted model, for example AfAm has a moderate U-shape effect. Note that the tick marks on the horizontal axis are the quantiles, that is, 0 per cent, 10 per cent, 20 per cent to 100 per cent. 
The interpretation of the partial dependence plots in Figure 5 provides an interesting snapshot of the post-Katrina situation in New Orleans. The first graph for AfAm reveals that as the percentage of African Americans in a census tract increases, so a

Figure 5 Variable importance (left) and partial dependence plots in MART model of flood depth

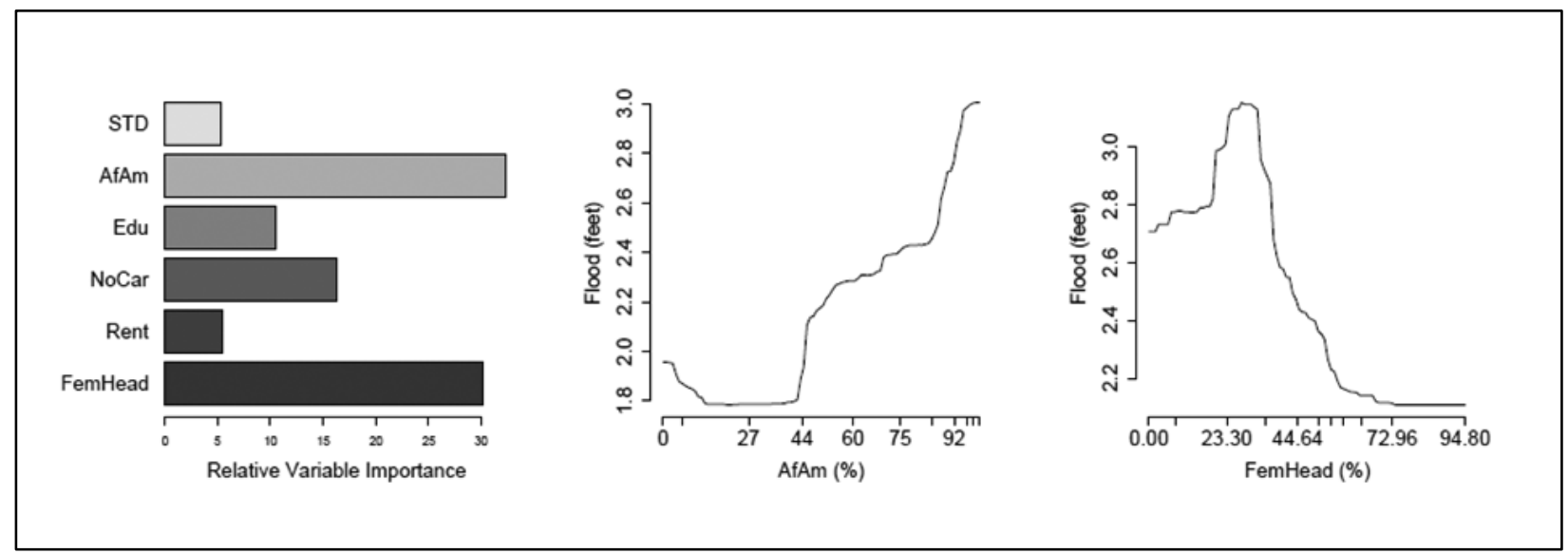

Source: Authors' elaboration of results

higher level of flooding was experienced. In other words African-American neighbourhoods received the deepest floodwaters. The plot also shows that census tracts with the lowest percentage of African Americans also experienced relatively high levels of flooding. This is understandable given the heavy damage experienced from the break of the 17th Street Canal in the predominantly white Lakeview area of the city. However, flood height in the highest percentage African-American census tracts exceeds those in the lowest percentage African-American census tracts.

FemHead, the second most important variable in the model, also displays a nonlinear effect as seen in Figure 5. However, unlike AfAm, the depth of flood waters rose as the percentage of FemHead in a census tract increased until approximately 30 per cent of all families were female headed. At this point the association between water depth and percentage of families begins to decrease.

\section{Modelling structural damage}

The MART model for severe building damage consists of 1,263 individual regression trees and explains approximately 42 per cent of variability. AfAm is clearly the most important variable in the model, with NoCar only having a moderate effect. Figure 6 shows that the partial dependence plot for AfAm is similar to the one in the flood depth model. Again, African-American neighbourhoods suffered the highest degree of building damage-indeed, as measured by this variable, damage that likely resulted in demolition and the potential loss of community. One difference between the two plots is the lack of an upturn in the tail representing census tracts with the lowest percentage of African Americans. One possible explanation for this could be the quality of building structure. As previously mentioned, areas of 
affluence and largely white populations suffered flooding due to the break in the 17th Street Canal levee. However, the degree of flooding does not necessarily equate to building resiliency. Many homes in the 17th Street Canal area were rebuilt from the flooded building shells. It is possible that for other areas of the city, poorer housing quality would result in a more linear relationship between degree of flooding and the resulting loss of building fecundity.

Figure 6 Variable importance (left) and partial dependence plot of AfAm in MART model of percentage of severe damage

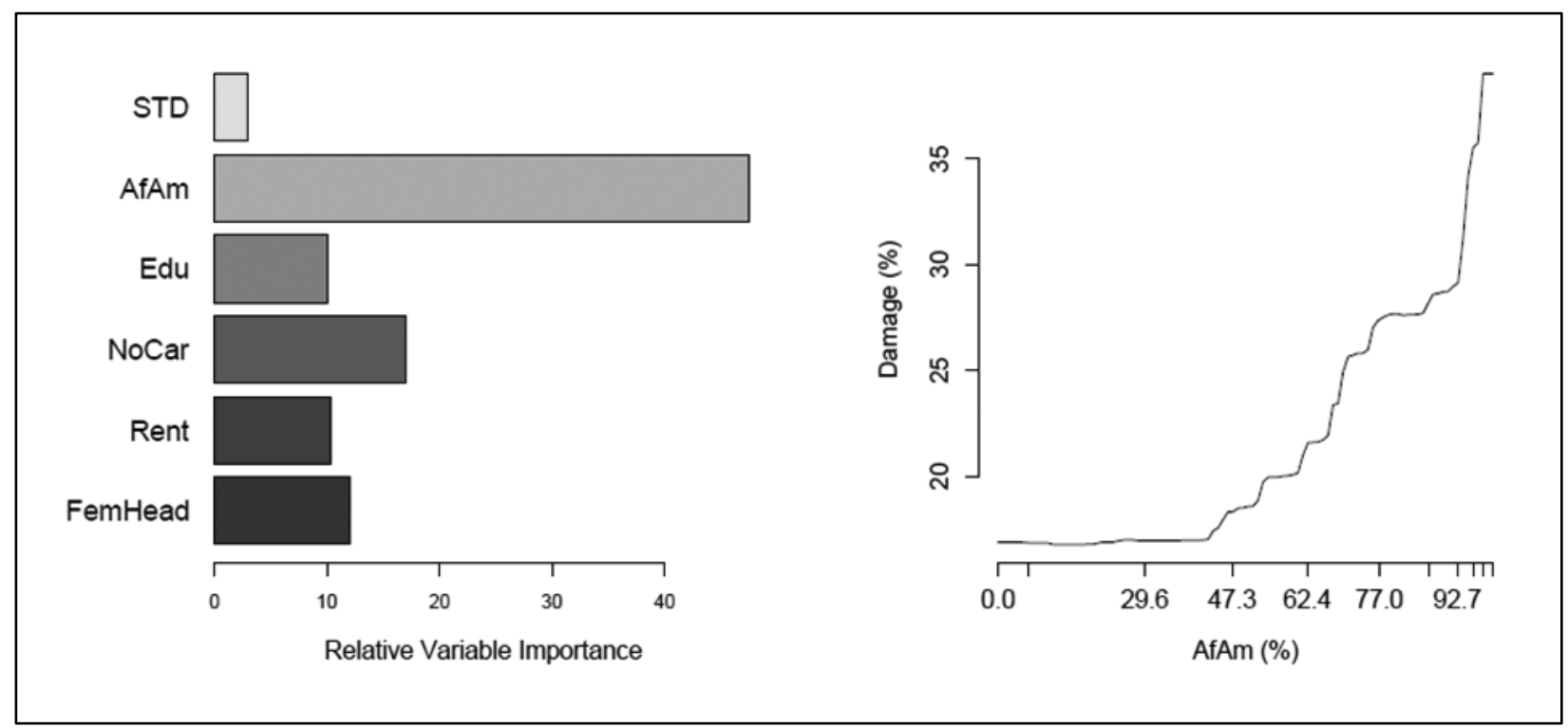

Source: Authors' elaboration of results.

\section{Modelling personal exposure through 911 calls}

Arguably the most interesting MART model is for 911 calls as this variable has not been previously used in analyses of this type, and because it captures personal exposure rather than just structural damage. This model consists of 908 individual regression trees and explains approximately one third of total variability. Figure 7 shows the variable importance and partial dependence plot for AfAm, which is again the most influential variable. As with the other models, NoCar and FemHead also have moderate importance, but for the first time STD also emerges as having 
Figure 7 Variable importance (left) and partial dependence plot of AfAm in MART model of 911 calls

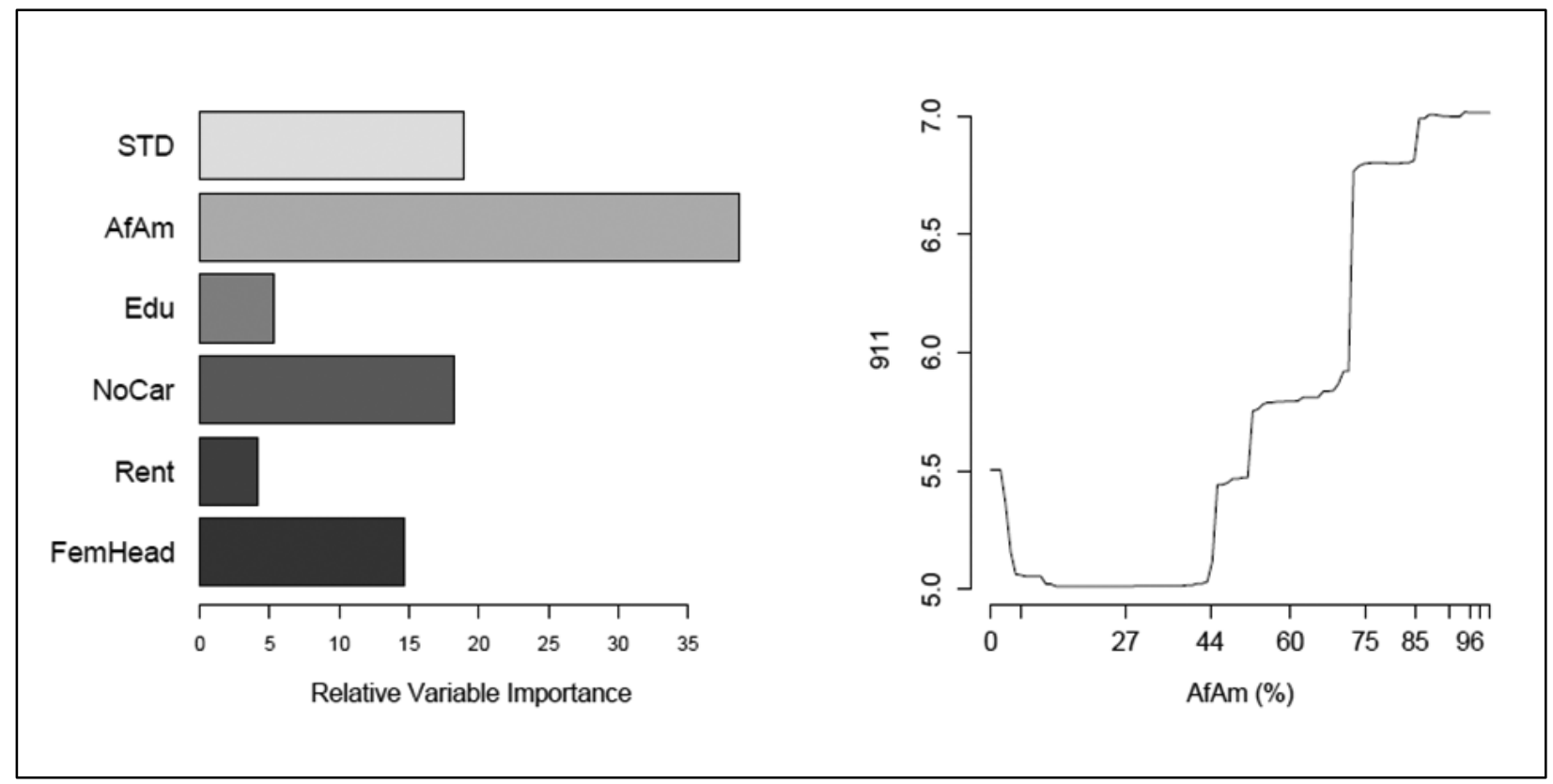

Source: Authors' elaboration of results.

moderate importance. The partial dependence plot for AfAm reflects a similar nonlinear curve to the MART flood model. In other words the number of 911 calls increased per census tract with higher percentages of African Americans. By referring back to Figure 5, it could be argued that this also mirrors the measures of structural exposure-the higher the percentage of African Americans in a census tract, the greater the damage from the storm, and the greater the need for help.

\section{Conclusions}

The human toll of a disaster in the United States is often counted in terms of immediate suffering. However, acuteness of exposure combined with pre-event health burden and the timeframe of recovery can extend this period of suffering into years or for generations. This paper has modelled three measures of exposure to Katrina: two structural measures (flood height and building damage), and one personal measure (phone calls made to emergency services). The analysis type chosen was MART, which can capture the non-additive behaviour among the social vulnerability regressors, and which has been shown to demonstrate sizable gains in explained variability over the more classical linear regression models. The relatively highunexplained variability among the responses can be explained by the geographical and social complexity of New Orleans. Nonetheless, MART has been successful in parsing out meaningful partial information with regards to measures influencing the potential future health burden, especially the impact African-American neighbourhoods experienced with all three measures of storm exposure. 
Katrina exposure increases as the percentage of African Americans in a census tract rises. Floodwaters were higher, building damage was greater, and there was more human suffering and need for rescue. This finding validates another descriptive analysis that has commented on the racial suffering in this catastrophe (Pastor et al., 2006). Many of the pre-Katrina conditions will have been exacerbated by this exaggerated exposure. As these results show, for many African-American neighbourhoods exposure was significantly high, resulting in the loss of homes and other important social connectors such as churches, which, when combined with financial constraints, helps explain why recovery has been so slow in occurring. In addition, the neighbourhoods that suffered the greatest exposure were already suffering from high rates of chronic and infectious disease, as well as poor birth outcomes, before the storm. This health vulnerability would have been exacerbated by the exposure, resulting in a greater health burden and a further impediment to recovery (Xiong et al., 2008). Of course, some of these stresses will also affect other racial groups, though as this study has shown, African-American neighbourhoods were hardest hit by all three exposure measures.

Further health vulnerability could also be interpreted from the results of Fem Head, which had an important impact on Flood and a moderate impact on the $\mathbf{9 1 1}$ model. As a result, census tracts with approximately 30 per cent of families being female headed and with children in the home suffered notable exposure during Katrina. For families of this type, there is often a heavy reliance on neighbouring friends and family. In other words, for neighbourhoods with a high proportion of African-American female-headed households there is a greater need for community (and social) capital. These neighbourhoods suffered high levels of storm exposure. Combining this exposure with a lack of financial buffering has resulted in the current (2008) environment of fragmented communities, where streets remain a mix of return and abandonment, and clinics, schools, stores and, maybe most importantly, churches remain closed. It is not surprising that the return to normalcy has been so slow to occur in these neighbourhoods.

Interestingly, Edu and Rent had limited impact in all of the exposure models. In an earlier version of the analysis performed on census block group level data for just one exposure measure, Flood, both Edu and Rent had a stronger influence suggesting a possible spatial scale effect. This indeed makes sense as subsequent research from the authors at the street level have identified marked spatial variation from street to street in terms of returnee, rental properties and abandonment (Curtis, Mills, Kennedy et al., 2007). From a modelling perspective, it would be interesting to see how MART results change with different spatial aggregations of data, both in terms of input variables and damage expressions.

Analysing data at this finer spatial resolution is obviously needed to target interventions and help re-establish community. However, at a broader level, when arguments are still being made as to why money should be leveraged, or why communities should be empowered to develop their own recovery agenda, the results of models as presented in this paper provide a useful foundation for understanding current 
impediments to recovery. Such a modelled foundation is also required if we are to start to understand the process of recovery from Katrina, and then expand this model to plan for future disasters.

\section{Endnotes}

1 Andrew Curtis is Visiting Associate Professor in the Department of Geography, University of Southern California, Los Angeles, United States; Bin Li is Assistant Professor in the Department of Experimental Statistics, Louisiana State University, Baton Rouge, United States; Brian D. Marx is Professor in the Department of Experimental Statistics, Louisiana State University, Baton Rouge, United States; Jacqueline W. Mills is Assistant Professor in the Department of Geography, California State University, Long Beach, United States; and John Pine is Director of the Research Institute for Environment, Energy and Economics, Appalachian State University, Boone, United States.

2 The catastrophe surrounding Hurricane Katrina involved both typical destruction associated with the storm and the subsequent failure of sections of the levee system. As a result the loss, or exposure, is partly due to a physical process and partly a result of human agency. This paper will encapsulate all of this destructive activity in the single use of the word 'Katrina', in the spirit of other holistic disaster analyses such as that of Erikson (1976).

\section{References}

Abramson, D. and R. Garfield (2006) On the Edge: Children and Families Displaced by Hurricanes Katrina and Rita Face a Looming Medical and Mental Health Crisis. Mailman School of Public Health, Columbia University, New York, NY.

Bourque, L.B., J.M. Siegel, M. Kano and M.M. Wood (2006) 'Weathering the storm: the impact of hurricanes on physical and mental health'. Annals of the American Academy. 604(1). pp. 129-151.

Breiman, L., J. Friedman, R. Olshen and C. Stone (1984) Classification and Regression Trees. Wadsworth, Belmont, CA.

Curtis, A. and M. Leitner (2006) Geographical Information Systems and Public Health: Eliminating Perinatal Disparity. Idea Group Inc Press, Hershey, PA.

Curtis, A., J.W. Mills, B. Kennedy, A.S. Fotheringham and T. McCarthy (2007) 'Incorporating a spatial video acquisition system into disaster response and recovery: a case study of the Lower $9^{\text {th }}$ Ward'. Journal of Contingencies and Crisis Management. 15(4). pp. 208-219. 
Curtis, A., J.W. Mills and M. Leitner (2007) 'Katrina and vulnerability: the geography of stress'. Journal of Health Care for the Poor and Underserved. 18(2). pp. 315-330.

Cutter, S.L., B.J. Boruff and W.L. Shirley (2003) 'Social vulnerability to environmental hazards'. Social Science Quarterly. 84(2). pp. 242-261.

DeSalvo, K.B. et al. (2007) 'Symptoms of posttraumatic stress disorder in a New Orleans workforce following Hurricane Katrina'. Journal of Urban Health. 84(2). pp. 142-152.

Dolan, M.A. and S.E. Krug (2006) 'Pediatric disasters preparedness in the wake of Katrina: lessons to be learned'. Clinical Pediatric Emergency Medicine. 7(1). pp. 59-66.

Dow, K. and S.L. Cutter (1998) 'Crying wolf: repeat responses to hurricane evacuation orders'. Coastal Management. 26(4). pp. 237-252.

Dow, K. and S.L. Cutter (2000) 'Public orders and personal opinions: household strategies for hurricane risk assessment'. Environmental Hazards. 2(4). pp. 143-155.

Dow, K. and S.L. Cutter (2002) 'Emerging hurricane evacuation issues: Hurricane Floyd and South Carolina'. Natural Hazards Review. 3(1). pp. 12-18.

Enarson, E. (2002) 'Through women's eyes: a gendered research agenda for disaster social science'. Disasters. 22(2). pp. 157-173.

Erikson, K.T. (1976) 'Disaster at Buffalo Creek: loss of communality at Buffalo Creek'. American Journal of Psychiatry. 133(3). pp. 302-305.

Friedman, J. (2001) 'Greedy function approximation: a gradient boosting machine'. The Annals of Statistics. 29(5). pp. 1189-1232.

Galea, S. et al. (2007) 'Exposure to hurricane-related stressors and mental illness after Hurricane Katrina'. Archives of General Psychiatry. 64(12). pp. 1427-1434.

Green, R., L. Bates and A. Smyth (2007) 'Impediments to recovery in New Orleans' Upper and Lower 9th Ward: one year after Hurricane Katrina'. Disasters. 31(4). pp. 311-335.

Greenough, P.G. et al. (2008) 'Burden of disease and health status among Hurricane Katrina-displaced persons in shelters: a population-based cluster sample'. Annals of Emergency Medicine. 51(4). pp. 426-432. 